\title{
Probing $U(1)_{B-L}$ model through the process $e^{+} e^{-} \rightarrow v_{\tau} \bar{v}_{\tau} \gamma$
}

\section{María A. Hernández-Ruiz ${ }^{* \dagger}$}

Unidad Académica de Ciencias Químicas, Universidad Autónoma de Zacatecas,

Apartado Postal 585, 98060 Zacatecas, México.

E-mail: mahernanduaz.edu.mo

\section{A. Gutiérrez-Rodríguez}

Facultad de Física, Universidad Autónoma de Zacatecas,

Apartado Postal C-580, 98060 Zacatecas, México.

E-mail: alexquefisica.uaz.edu.mx

We probe the $U(1)_{B-L}$ model via the process $e^{+} e^{-} \rightarrow v_{\tau} \bar{v}_{\tau} \gamma$. We also analyzed the effects of extra gauge boson $Z^{\prime}$ in the cross section at high energy linear $e^{+} e^{-}$collider and high luminosity; namely, International Linear Collider (ILC) and Compact Linear Collider (CLIC).

The European Physical Society Conference on High Energy Physics

22-29 July 2015

Vienna, Austria

\footnotetext{
${ }^{*}$ Speaker.

†The author thanks The Universidad Autónoma de Zacatecas and SNI-CONACyT México.
} 


\section{Introduction}

It is possible to study some phenomenological features associated with an extra neutral gauge boson by considering a minimal $B-L$ (baryon number minus lepton number) model [四. The $B-L$ symmetry plays an important role in various physics scenarios beyond the Standard Model, for example, the gauge $U(1)_{B-L}$ symmetry group is contained in a GUT described by a $S O(10)$ group. The $B-L$ model is attractive due to its relatively simple theoretical structure, and the crucial test of the model is the detection of the new heavy neutral $\left(Z^{\prime}\right)$ gauge boson [1, 目, 田]. The analysis of precision electroweak measurements indicates that the new $Z^{\prime}$ gauge boson should be heavier than about 1.2 TeV. On the other hand, recent bounds from the LHC indicate that the $Z^{\prime}$ gauge boson should be heavier than about $2 \mathrm{TeV}$ [ [5, 目], while future LHC runs at 13-14 TeV could increase the $Z^{\prime}$ mass bounds to higher values, or may be lucky and find evidence for its presence. Further studies of the $Z^{\prime}$ properties will require a new linear collider.

Our aim in the present work is to analyze the reaction $e^{+} e^{-} \rightarrow v_{\tau} \bar{v}_{\tau} \gamma$ in the framework of a $U(1)_{B-L}$ model. We study the indirect effects of extra gauge bosons in the cross sections of the process $e^{+} e^{-} \rightarrow v_{\tau} \bar{v}_{\tau} \gamma$ at high energy linear $e^{+} e^{-}$colliders; namely, International Linear Collider (ILC) [U] and Compact Linear Collider (CLIC) [8]]. In addition to the limits from hadron colliders, an improvement on the sensitivity of the physical observables will be reached at future $e^{+} e^{-}$linear collider. Finally, we discuss how accurately the $U(1)_{B-L}$ model parameters will be measurable at the ILC and CLIC.

\section{Cross section of the process $e^{+} e^{-} \rightarrow v_{\tau} \bar{v}_{\tau} \gamma$ in the $U(1)_{B-L}$ model}

We calculate the cross section via the process $e^{+} e^{-} \rightarrow v_{\tau} \bar{v}_{\tau} \gamma$ in the context of the $U(1)_{B-L}$ model at future high-energy and high luminosity linear electron-positron colliders, such as the ILC and CLIC. The Feynman diagrams contributing to the process are shown in Figure 1. The expressions for the total cross section of the process for the different contributions, can be written in the following form

$$
\sigma_{\text {tot }}\left(e^{+} e^{-} \rightarrow v_{\tau} \bar{v}_{\tau} \gamma\right)=\sigma_{1,3}+\sigma_{2,4}+\sigma_{\text {int }}
$$

where

$$
\begin{aligned}
\sigma_{1,3}= & \int\left(\frac{G_{F}^{2} \alpha}{2 \pi^{2}}\right) \frac{M_{Z}^{4}\left(g_{V}^{2}+g_{A}^{2}\right)}{\left[\left(M_{Z}^{2}-s+2 \sqrt{s} E_{\gamma}\right)^{2}+M_{Z}^{2} \Gamma_{Z}^{2}\right]} \\
& \times\left[\frac{s-2 \sqrt{s} E_{\gamma}+2 E_{\gamma}^{2}-E_{\gamma}^{2} \sin ^{2} \theta_{\gamma}}{\sqrt{s} E_{\gamma} \sin ^{2} \theta_{\gamma}}\right]\left(\sqrt{s}-2 E_{\gamma}\right) d E_{\gamma} d \cos \theta_{\gamma} \\
\sigma_{2,4}= & \int\left(\frac{G_{F}^{2} \alpha}{2 \pi^{2}}\right) \frac{M_{Z^{\prime}}^{4}\left(g^{\prime 2}+g_{A}^{\prime 2}\right)}{\left[\left(M_{Z^{\prime}}^{2}-s+2 \sqrt{s} E_{\gamma}\right)^{2}+M_{Z^{\prime}}^{2} \Gamma_{Z^{\prime}}^{2}\right]} \\
& \times\left[\frac{s-2 \sqrt{s} E_{\gamma}+2 E_{\gamma}^{2}-E_{\gamma}^{2} \sin ^{2} \theta_{\gamma}}{\sqrt{s} E_{\gamma} \sin ^{2} \theta_{\gamma}}\right]\left(\sqrt{s}-2 E_{\gamma}\right) d E_{\gamma} d \cos \theta_{\gamma}
\end{aligned}
$$




$$
\begin{aligned}
\sigma_{i n t}= & \int\left(\frac{G_{F}^{2} \alpha}{\pi^{2}}\right) \frac{2\left(g_{V} g_{V}^{\prime}+g_{A} g_{A}^{\prime}\right)}{\left[\left(M_{Z}^{2}-s+2 \sqrt{s} E_{\gamma}\right)^{2}+M_{Z}^{2} \Gamma_{Z}^{2}\right]} \\
& \times \frac{\left[\left(M_{Z}^{2}-s+2 \sqrt{s} E_{\gamma}\right)\left(M_{Z^{\prime}}^{2}-s+2 \sqrt{s} E_{\gamma}\right)+M_{Z} M_{Z^{\prime}} \Gamma_{Z} \Gamma_{Z^{\prime}}\right]}{\left[\left(M_{Z^{\prime}}^{2}-s+2 \sqrt{s} E_{\gamma}\right)^{2}+M_{Z^{\prime}}^{2} \Gamma_{Z^{\prime}}^{2}\right]} \\
& \times\left[\frac{s-2 \sqrt{s} E_{\gamma}+2 E_{\gamma}^{2}-E_{\gamma}^{2} \sin ^{2} \theta_{\gamma}}{\sqrt{s} E_{\gamma} \sin ^{2} \theta_{\gamma}}\right]\left(\sqrt{s}-2 E_{\gamma}\right) d E_{\gamma} d \cos \theta_{\gamma}
\end{aligned}
$$

where

$$
\begin{aligned}
g_{V}^{f} & =T_{3}^{f} \cos \theta_{B-L}-2 Q_{f} \sin ^{2} \theta_{W} \cos \theta_{B-L}+\frac{2 g_{1}^{\prime}}{g} \cos \theta_{W} \sin \theta_{B-L}, \\
g_{A}^{f} & =T_{3}^{f} \cos \theta_{B-L}, \\
g_{V}^{\prime} & =-T_{3}^{f} \sin \theta_{B-L}-2 Q_{f} \sin ^{2} \theta_{W} \sin \theta_{B-L}+\frac{2 g_{1}^{\prime}}{g} \cos \theta_{W} \cos \theta_{B-L}, \\
g_{A}^{\prime} & =-T_{3}^{f} \sin \theta_{B-L},
\end{aligned}
$$

here $g=e / \sin \theta_{W}$ and $\theta_{B-L}$ is the $Z-Z^{\prime}$ mixing angle. The current bound on this parameter is $\left|\theta_{B-L}\right| \leq 10^{-3}[\theta]$. In the decoupling limit, that is to say, when $g_{1}^{\prime}=0$ and $\theta_{B-L}=0$ the couplings of the standard model (SM) are recovered.



(1)



(3)

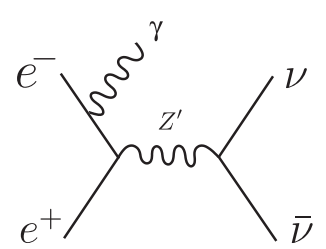

(2)

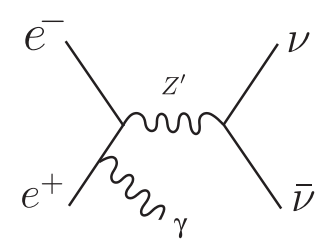

(4)

Figure 1: The Feynman diagrams contributing to the process $e^{+} e^{-} \rightarrow v_{\tau} \bar{v}_{\tau} \gamma$ in the $U(1)_{B-L}$ model.

\section{Results and Conclusion}

We evaluate the total cross section of the process $e^{+} e^{-} \rightarrow v_{\tau} \bar{v}_{\tau} \gamma$ in the context of the $B-L$ model at next generation linear $e^{+} e^{-}$colliders such as the ILC and CLIC. Using the following values for numerical computation [Q] : $\sin ^{2} \theta_{W}=0.23126 \pm 0.00022, M_{Z}=91.1876 \pm 0.0021 \mathrm{GeV}$, $\Gamma_{Z}=2.4952 \pm 0.0023 \mathrm{GeV}$, and considering the most recent limit from LEP [Шర]]: $\frac{M_{Z^{\prime}}}{g_{1}^{\prime}} \geq 7 \mathrm{TeV}$, in 
our numerical analysis, we obtain the total cross section thus, in our numerical computation, we will assume $\sqrt{s}, M_{Z^{\prime}}$ and $g_{1}^{\prime}$ as free parameters.

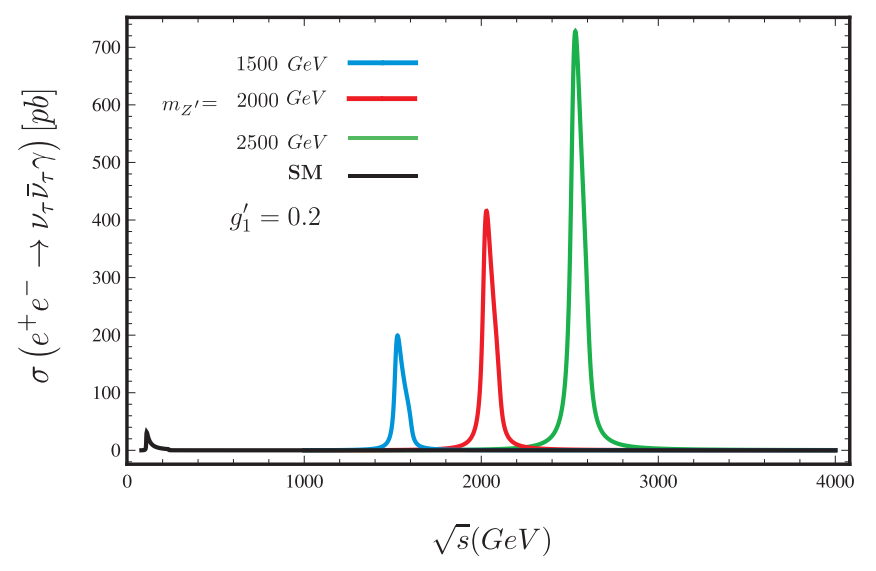

Figure 2: The total cross section of the production processes $\sigma_{t o t}\left(e^{+} e^{-} \rightarrow v_{\tau} \bar{v}_{\tau} \gamma\right)$ as a function of the collision energy for fixed value $g_{1}^{\prime}=0.2$ and $\left|\theta_{B-L}\right| \leq 10^{-3}$. The curves are for SM (black) and $M_{Z^{\prime}}=1500$, 2000, $2500 \mathrm{GeV}$ (blue, red, green). The resonance corresponds to the $Z^{\prime}$ new gauge boson.

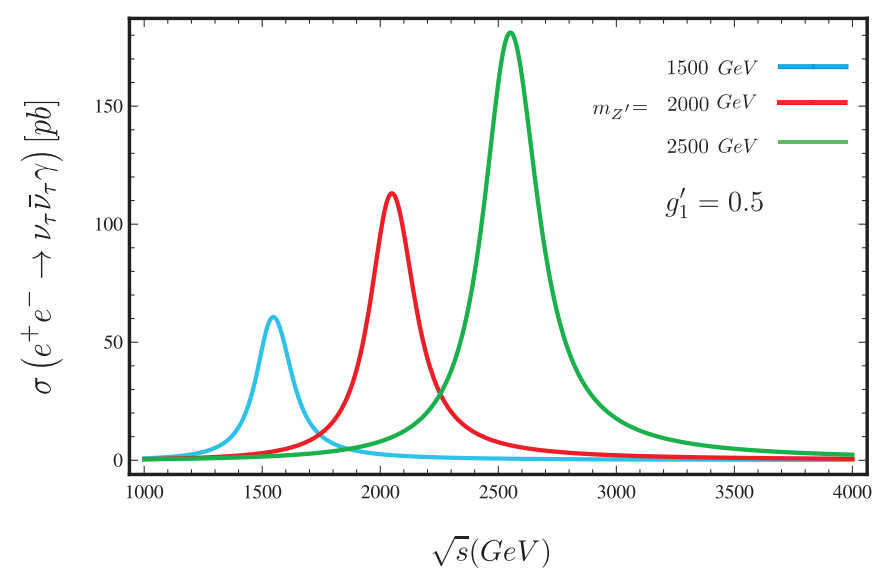

Figure 3: The total cross section of the production processes $\sigma_{t o t}\left(e^{+} e^{-} \rightarrow v_{\tau} \bar{v}_{\tau} \gamma\right)$ as a function of the collision energy for fixed value $g_{1}^{\prime}=0.5$ and $\left|\theta_{B-L}\right| \leq 10^{-3}$. The curves are for $M_{Z^{\prime}}=1500,2000,2500$ $\mathrm{GeV}$ (blue, red, green). The resonance corresponds to the $Z^{\prime}$ new gauge boson.

In conclusion, we find that the future linear $e^{+} e^{-}$collider experiments such as the ILC and CLIC could test the $U(1)_{B-L}$ model, and it would be possible to perform precision measurements of the $Z^{\prime}$ gauge boson, as well as of the parameters of the model: $\theta_{B-L}$ the $Z-Z^{\prime}$ mixing angle and $g_{1}^{\prime}$, complementing other studies on the $U(1)_{B-L}$ model. The SM expression for the cross section of the reaction $e^{+} e^{-} \rightarrow v_{\tau} \bar{v}_{\tau} \gamma$ can be obtained in the decoupling limit, that is to say, when $\theta_{B-L}=0$ and $g_{1}^{\prime}=0$, in this case the terms that depend on $\theta_{B-L}$ and $g_{1}^{\prime}$ are zero and the equation (LD) is reduced to the expression given for the standard model. In addition, the analytical and numerical results for the total cross section have never been reported in the literature. 


\section{References}

[1] L. Basso, A. Belyaev, S. Moretti and C. H. Shepherd-Themistocleous, "Phenomenology of the minimal B-L extension of the Standard model: Z' and neutrinos," Physical Review, D80, 055030 (2009), arXiv:0812.4313 [hep-ph].

[2] A. Gutiérrez-Rodríguez and M. A. Hernández-Ruiz, " $Z$ ' resonance and associated $Z h$ production at future Higgs boson factory: ILC and CLIC", Advances in High Energy Physics 2015, 593898 (2015), arXiv:1506.07575 [hep-ph].

[3] A. González-Sánchez, A. Gutiérrez-Rodríguez, and M. A. Hernández-Ruiz, "Bounds on the Number of Light Neutrinos Species, $g_{1}^{\prime}$ Coupling and $Z-Z^{\prime}$ Mixing Angle in a $U(1)_{B-L}$ Model", Journal of Modern Physics 6, 1077 (2015).

[4] A. Gutiérrez-Rodríguez, "Proceedings, 20th International Conference on Particles and Nuclei (PANIC 14): Hamburg, Germany, August 24-29, 2014”, DESY-PROC-2014-04. INSPIRE-1375563.

[5] ATLAS Collaboration, "Search for high-mass dilepton resonances in 20/fb of pp collisions at $\sqrt{s}=8$ TeV with the ATLAS experiment", ATLAS-CONF-2013-017.

[6] CMS Collaboration, "Search for resonances in the dilepton mass distribution in pp collisions $\sqrt{s}=8$ TeV”, Tech. Rep. CMS-PAS-EXO-12-061, CERN, 2013.

[7] R. Brinkmann et al., TESLA technical design report, DESY-2001-011, 2001; G. A. Loew, Report from the International Linear Collider Technical Review Committe SLAC-PUB-10024, 2003.

[8] R. W. Assmann et al., The CLIC Study Team, A $3 \mathrm{TeV} e^{+} e^{-}$linear collider based on CLIC technology, CERN-2003-007, Geneva, 2003.

[9] K. A. Olive, K. Agashe, et al., Review of particle physics, Chinese Physics C38, 090001 (2014).

[10] G. Cacciapaglia, C. Csaki, G. Marandella and A. Strumia, Physical Review D74, 033011 (2006). 